\title{
2 Lexikalische Repräsentation von Valenz
}

Gegenstand der nachfolgenden Untersuchungen sind lexikalisch lizenzierte Komplementweglassungen, d. h. solche, die durch die Verbwahl beschränkt sind. Beispiele wie in (1) zeigen, dass ,null complementation, while motivated by such pragmatic drives as effort conservation, is linguistically constrained“ (Ruppenhofer 2018: 204). Während manche Verben Komplementweglassungen problemlos erlauben (z. B. essen), blockieren andere, semantisch ähnliche sie unter sonst gleichen Bedingungen (z. B. verspeisen).

Vor dem Hintergrund solch lexikalischer Idiosynkrasien wird die Option, ein Komplement unrealisiert zu lassen, in Standarddarstellungen zum Deutschen an der Valenz des jeweiligen Verbs festgemacht. Für ältere Valenzkonzepte waren weglassbare Komplemente noch problematisch, da Valenz lange Zeit als einheitliches Phänomen betrachtet wurde - nämlich als die Fähigkeit von bestimmten sprachlichen Ausdrücken, insbesondere Verben ${ }^{36}$, Ergänzungen zu verlangen. Mit diesem Valenzbegriff einher ging der Versuch, Ausdrücke, die von dieser Ergänzungsbedürftigkeit betroffen sind, den sog. Ergänzungen, von Ausdrücken, die von dieser Ergänzungsbedürftigkeit nicht betroffen sind, den sog. Angaben, zu unterscheiden (vgl. Welke 2011: 45). Es wurden zahlreiche zumeist monokriteriale Lösungsansätze mit entsprechenden operationalisierten Testverfahren entwickelt. Die Darstellungen in den Kapiteln 2.1 und 2.2 werden zeigen, dass die eindimensionale Ausrichtung dieser Ansätze, d.h. der Versuch, Verbbegleitern die Eigenschaft valenzgebunden aufgrund genau einer Eigenschaft zuzuweisen, mit zahlreichen Problemen verbunden ist. Es zeigt sich, dass Verben ganz unterschiedliche Forderungen an ihre jeweiligen Begleiter stellen und dass diese nicht zwangsläufig miteinander korrelieren.

Im Laufe der Zeit hat sich eine multidimensionale Auffassung von Valenz entwickelt. In einem solchen Valenzkonzept, wie es von Jacobs (1992a, 1994a) umrissen und verschiedentlich aufgegriffen und weiterentwickelt wurde (vgl. z. B. Blume (1993), (2000); Engelberg (2000); Jacobs (2003)), wird Valenz nicht mehr als einheitliches Phänomen betrachtet, sondern als eine Art cover term für verschiedene voneinander unabhängige Valenzbindungsrelationen formaler und

36 „Die Valenztheorie begann dort, wo man Valenz am unmittelbarsten beobachten kann, bei den Verben.“ (Welke 2011: 45) Im Zuge der Entwicklung ergab sich dann die Tendenz, den Valenzbegriff auch auf andere Wortarten - etwa Substantive und Adjektive - auszuweiten. Eine überblicksartige Darstellung der einzelnen Valenzträger findet sich u. a. bei Welke (2011: 106ff.) und Sommerfeldt (2006). Detailliertere Darstellungen etwa zur Substantivvalenz finden sich u. a. bei Teubert (1979), Sommerfeldt/Schreiber (1977), Bassola (2003) und Hölzner (2007), Ausführungen zur Adjektivvalenz finden sich u. a. bei Herbst (1983) und Sommerfeldt/Schreiber (1974).

๖ Open Access. ( 2021 Tanja von der Becke, publiziert von De Gruyter. (c) BY Dieses Werk ist lizensiert unter einer Creative Commons Namensnennung 4.0 International Lizenz.

https://doi.org/10.1515/9783110726268-002 
semantischer Art (vgl. Ágel/Fischer 2010: 268). Valenz wird also als vielschichtiges Phänomen betrachtet, das auf semantischer und kategorialer Ebene zu beschreiben ist, wobei die verschiedenen Valenzbindungsbeziehungen unabhängig voneinander im Lexikoneintrag eines Verbs zu verzeichnen sind. Auch im Rahmen dieser Arbeit wird eine multidimensionale Auffassung von Valenz vertreten. Grundlage bildet die von Jacobs entwickelte Multidimensionale Valenztheorie (MVT), wobei von der aktuellen Version ausgegangen wird, „die sich von früheren Versionen zwar nicht in den Grundgedanken, aber in wichtigen Details unterscheidet“ (Jacobs 2003: 378). Die zentralen Ideen dieses Konzepts werden in Kapitel 2.3 dargestellt.

Die theoretische Brisanz der Zerlegung von Valenz in verschiedene (semantische und kategoriale) Valenzbeziehungen liegt darin, dass die einzelnen Relationen als voneinander unabhängig betrachtet werden. „Es handelt sich also nicht um verschiedene Aspekte ein und desselben Phänomens, die immer zusammen vorliegen müssen, sondern um verschiedene Phänomene, die zusammen vorliegen können [...].“ (ebd. 390) Hierbei lassen sich „Fälle, in denen es zu einer globalen Divergenz von kategorialer und semantischer Valenz kommt, als auch solche, in denen Dimensionen der jeweils gleichen Valenzebene nicht konvergieren“ unterscheiden (ebd.). Der wohl umstrittenste Fall - in der Valenzforschung unter der Bezeichnung fakultative Ergänzungen intensiv und kontrovers diskutiert -, bei dem Merkmals- ohne Realisierungsforderungen vorzuliegen scheinen, wird in Kapitel 2.4 dargestellt und in Kapitel 5 auf Grundlage eigener Untersuchungen speziell unter dem Gesichtspunkt semantischer Spezialisierungen impliziter Argumente kritisch diskutiert. In Kapitel 6 schließen sich die die Hauptuntersuchungen ergänzenden Überlegungen zur definiten und indefiniten (bzw. definitheitsneutralen) Interpretation impliziter Argumente an.

\subsection{Grundzüge der Valenztheorie}

Die moderne Valenztheorie, die seit Jahren immer wieder im Fokus linguistischer Untersuchungen steht ${ }^{37}$, ist vergleichsweise jung; sie wird maßgeblich auf den Franzosen Lucien Tesnière (1893 - 1954) zurückgeführt. Der Valenzgedanke als solcher - also die Idee, dass bestimmte sprachliche Ausdrücke, insbesondere

37 Regelmäßig kommt es bei linguistischen Untersuchungen zur Valenz zu Neuerungen etwa in Form von Präzisierungen, Einwänden oder Gegenentwürfen, so dass die immer umfangreichere Valenzliteratur heutzutage nur noch schwer zu überschauen ist. Waren in Schumachers Valenzbibliographie (1988) noch 2377 Valenzarbeiten verzeichnet, schätzt Ágel die Anzahl der Valenzbeiträge im Jahr 2000 bereits auf mehr als 3000. 
Verben, sich nur mit bestimmten anderen Wörtern verbinden und nach einer bestimmten Anzahl von Begleitern verlangen - ist jedoch sehr viel älter ${ }^{38}$. Erste Vorläuferkonzepte finden sich bereits in der Antike (vgl. Seidel 2003), in der arabischen grammatischen Tradition (vgl. Ownes 2003) und bei den mittelalterlichen Modisten (vgl. Seppänen 2003). Valenzaspekte werden hier zwar noch nicht direkt angesprochen; Aussagen in verschiedenen Kontexten belegen jedoch, dass Grundgedanken der Valenztheorie auch damals schon erkannt und berücksichtigt wurden.

Als unmittelbare Wegbereiter der Valenztheorie gelten u. a. Meiner (1781/1971), dessen Versuch einer an der menschlichen Sprache abgebildeten Vernunftslehre „einen Meilenstein (auch) in der Geschichte der Valenzidee darstellt“ (Ágel 2000: 21), und auch Bühler, der in diesem Zusammenhang vor allem mit folgender Passage zitiert wird:

Es bestehen in jeder Sprache Wahlverwandtschaften; das Adverb sucht sein Verbum und ähnlich die anderen. Das läßt sich auch so ausdrücken, daß die Wörter einer bestimmten Wortklasse eine oder mehrere Leerstellen um sich herum eröffnen, die durch Wörter bestimmter anderer Wortklassen ausgefüllt werden müssen.

(Bühler 1999³ [1934]: 173)

Weder Bühler selbst noch ein anderer Wissenschaftler verfolgen diese Idee jedoch weiter (vgl. Engel 2006). So bleiben Bemerkungen dieser Art zunächst Ansätze, die kaum systematisiert werden (vgl. Helbig 1989: 208). Erst Tesnière gelingt es dann in den 50er Jahren des letzten Jahrhunderts, die Valenzidee so zu formalisieren, dass sie in einen abgeschlossenen theoretischen Rahmen überführt werden konnte. „Folglich ist ,Valenz bei Tesnière keine bloße intuitive Idee mehr, sondern auch eine theoretische Notwendigkeit, die sich aus dem Grammatikmodell, seiner Dependenzgrammatik, ergibt.“ (Ágel 2000: 32)

Diese umfassende Dependenzgrammatik ${ }^{39}$ hat Tesnière in seiner erst posthum veröffentlichten Arbeit Élements des syntaxe structurale ${ }^{40}$ beschrieben. Darin analysiert er Sätze nicht als lineare Abfolge von Wörtern, sondern als komplexe hierarchisch organisierte Abhängigkeitsbeziehungen (sog. Konnexionen),

38 Eine detaillierte Darstellung der umfassenden Vorgeschichte der Valenzidee findet sich u. a. in Ágel (2000: 7 - 82) sowie in dem zweibändigen Handbuch „Dependenz und Valenz“ (2003, 2006). 39 Eine vollständige Erläuterung der Dependenzgrammatik von Tesnière wird hier nicht angestrebt. Der interessierte Leser sei daher auf eine überblicksartige Darstellung der Tesnièreschen Dependenzgrammatik bei Ágel (2000: 33ff.) sowie auf ausführlichere Beschreibungen u. a. bei Heringer (1993) und Weber (1997²) verwiesen. Ein ausführlicher Bericht zum Forschungsstand findet sich u. a. in dem zweibändigen Handbuch „Dependenz und Valenz“ (2003, 2006).

40 Die Darstellungen dieser Arbeit beziehen sich ausschließlich auf das 1959 posthum veröffentlichte Hauptwerk von Tesnière, Élements des syntaxe structurale, bzw. auf die 1980 erschienene deutsche Übersetzung von Engel, Grundzüge der strukturalen Syntax. 
darstellbar in einem konkreten oder virtuellen Baumdiagramm ${ }^{41}$ („Stemma“). Das Verb steht dabei auf der höchsten Ebene der Dependenzordnung; es bildet den obersten Knoten - also das Satzzentrum -, von dem alle Konstituenten des Satzes mittelbar oder unmittelbar abhängen (vgl. Hoffmann 2010³: 580f.).

Der Konzeptualisierung der Aktanten- und Valenzproblematik dienen bei Tesnière dann vor allem zwei Metaphern aus dem nichtgrammatischen Bereich (vgl. Askedal 2003: 90). Ausgehend von seiner berühmten Dramen-Metapher befasst sich Tesnière zunächst mit der zentralen Satzkonstitution durch das Verb und führt die Unterscheidung zwischen Aktanten (Ergänzungen) und Zirkumstanten (Angaben) ein. Er schreibt:

Der verbale Nexus, der bei den meisten europäischen Sprachen im Zentrum steht, läßt sich mit einem kleinen Drama vergleichen. Wie das Drama umfaßt er notwendig ein Geschehen und meist auch noch Akteure und Umstände.

(Tesnière 1980: 93)

Tesnière vergleicht in dieser Metapher den in einem Satz beschriebenen Sachverhalt mit einer auf der Bühne dargestellten Szene eines Dramas. Die Szene umfasst das Drama-Geschehen, welches - übertragen auf den Satz - dem Verb entspricht. Darüber hinaus sind an einer Szene Schauspieler beteiligt, die eine bestimmte Rolle übernehmen. Diese Aufgabe erfüllen in einem Satz die sog. Ergänzungen, die aufgrund ihrer Beteiligung am Geschehen auch als Mitspieler oder Aktanten bezeichnet werden. Des Weiteren wird die Szene durch Zirkumstanten ergänzt, die die Kulisse bildlich, räumlich, zeitlich oder in anderer Hinsicht bestimmen. Die Zirkumstanten geben also die näheren Umstände des Geschehens an und werden daher auch als Angaben bezeichnet (vgl. Pittner/Berman 2007²: 43).

Das traditionelle (aristotelische) Prinzip der Binarität von Subjekt und Prädikat wird bei Tesnière durch das Prinzip der endozentrischen Verbzentriertheit ersetzt: Die Satzstruktur entfaltet sich aus dem lexikalischen Verb heraus - das Verb ist also Voraussetzung für die Satzstruktur. Diese Verbzentriertheit begründet „eine funktionale Dreiteilung des Satzes - verbaler Valenzträger, Aktant, Angabe“ (Ágel/Fischer 2010: 257), wobei jedoch - ähnlich wie die Akteure und Umstände eines Dramas - Aktanten und Angaben keine gleichwertigen Partizipanten eines Satzes sind. Für einen Satz sind nur das Verb sowie seine Aktanten konstitutiv, wohingegen ,die Angaben im Satz eine externe, die konstitutiven Partizipanten rahmende Funktion“ haben (ebd.). Während Angaben zum Verb also - im Sinne Tesnières Dependenzgrammatik - ausschließlich in einer Dependenzbeziehung zueinander stehen, existiert zwischen Aktanten und

41 Konkrete oder reale Stemmata enthalten konkrete Wörter, wohingegen virtuelle Stemmata nur Symbole für die Wortarten enthalten. 
Verb eine zusätzliche Valenzrelation. Diese führt Tesnière mit Hilfe einer weiteren Struktur-Metapher ein. Als Ausgangspunkt dient hierbei die Übernahme einer vereinfachten Darstellung der aus der Chemie entlehnten Atomstruktur ${ }^{42}$ :

Man kann [...] das Verb mit einem Atom vergleichen, an dem Häkchen angebracht sind, so daß es - je nach Anzahl der Häkchen - eine wechselnde Zahl von Aktanten an sich ziehen und in Abhängigkeit halten kann. Die Anzahl der Häkchen, die ein Verb aufweist, und dementsprechend die Anzahl der Aktanten, die es regieren kann, ergibt das, was man die Valenz des Verbs nennt.

(Tesnière 1980: 161)

Hinter Tesnières Atom-Metapher steht die Idee, dass das Verb nicht einfach nur das strukturelle Zentrum des Satzes darstellt, sondern darüber hinaus auch die Fähigkeit besitzt, eine bestimmte Anzahl von Leerstellen zu eröffnen. Valenz $z^{43}$ bezeichnet bei Tesnière also „die Anzahl der potenziellen, in der Bedeutung des Verbs verankerten Aktanten eines verbalen Valenzträgers“ (Ágel/Fischer 2010: 257). Es lassen sich grundsätzlich avalente (Verben ohne Aktanten: Es friert.), monovalente (Verben mit einem Aktanten: Paula lacht.), divalente (Verben mit zwei Aktanten: Henri singt ein Lied.) und trivalente Verben (Verben mit drei Aktanten: Heinrich schenkt Lisa ein Buch.) unterscheiden. Die jeweilige Zuordnung gilt nach Tesnière auch dann, wenn nicht alle potenziellen Aktanten eines Verbs in einem Satz tatsächlich realisiert werden ${ }^{44}$. So gilt etwa das Verb essen als zweiwertig, unabhängig davon, ob es mit (Alfred isst einen Apfel) oder ohne Realisierung des zweiten Aktanten (Alfred isst) in einem Satz verwendet wird.

ImZentrum des Tesnièreschen Valenzbegriffs steht die Anzahl der potenziellen Aktanten eines verbalen Valenzträgers. Diese rein quantitative Valenz verknüpft Tesnière „mit der semantischen Funktion und der morpho-syntaktischen Realisie-

42 Eine ausführlichere Darstellung der Wertigkeitsmetapher sowie möglicher Kontaktpunkte zwischen natur- und geisteswissenschaftlichen Sicht- und Denkweisen finden sich bei Pongé (2003).

43 In der Chemie wurde die Valenztheorie Mitte des 19. Jahrhunderts begründet. Valenz bezeichnet hier „die rein quantitative Eigenschaft eines Atoms (eines bestimmten chemischen Elements), eine bestimmte Anzahl von Wasserstoffatomen zu binden“ (Lehmann 1992: 436). Tesnière führt dann den in der Chemie gebräuchlichen Terminus Valenz in die Sprachwissenschaft ein, indem er das Verb mit einem Atom vergleicht. So wie ein Atom Elemente an sich bindet, bindet das Verb eine bestimmte Anzahl von Aktanten an sich.

Verschiedentlich wurde in der Literatur angemerkt, dass bereits De Groot $\left(1949^{2}\right)$ den Begriff der (syntaktischen) Valenz verwendet (vgl. z. B. Lehmann 1992: 2). Es ist jedoch davon auszugehen, dass Tesnière sein 1959 posthum herausgegebenes Werk Éléments de syntaxe structurale in Manuskriptform schon zu Beginn der 40er Jahre fertiggestellt hatte (vgl. u.a. Kotschi (2001: 328), Engel (2006)).

44 So merkt Tesnière an, dass Verben nicht immer mit all ihren Aktanten vorkommen müssen (vgl. Tesnière 1980: 161). Der Frage, welche Faktoren die Sättigung der einzelnen Valenzstellen der verbalen Valenzträger determinieren, ging er allerdings nicht nach. 
rung der Aktanten (qualitative Valenz)“ (Ramers 2007²: 81). Als potenzielle valenzgeforderte Aktanten eines verbalen Valenzträgers kommen für ihn ausschließlich nominale Elemente in Frage (vgl. Tesnière 1980: 93). Dabei steht - in einem Aktivsatz - der Erstaktant im Nominativ und bezeichnet denjenigen, der eine Handlung ausführt; er entspricht damit dem traditionellen Subjekt (ebd. 100). Der Zweitaktant hingegen bezeichnet denjenigen, dem eine Tätigkeit/Handlung widerfährt; er entspricht dem direkten Objekt (Akkusativobjekt) (ebd.). Der Drittaktant steht im Dativ und bezeichnet den Nutznießer/Geschädigten einer Handlung; in ihm lässt sich das traditionelle indirekte Objekt (Dativobjekt) wiedererkennen (ebd. 101). Nicht-valenzgeforderte, die näheren Umstände eines Geschehens bezeichnende Angaben hingegen werden nach Tesnière - im Sinne seiner verallgemeinerten Dependenz-Hierarchie der Wortarten - als Adverbien realisiert (vgl. ebd. 94). Sie sind seiner Auffassung nach grundsätzlich fakultativ und können somit frei und in zahlenmäßig nicht festgelegter Weise hinzugefügt oder weggelassen werden.

Tesnières Verdienst, den Valenzgedanken - d.h. die Idee, dass Verben eine bestimmte Anzahl von Leerstellen eröffnen, die mit anderen sprachlichen Ausdrücken gefüllt werden müssen - fest in der Grammatikforschung zu etablieren, ist unbestritten. Seine Annahme, dass sich die valenzgeforderten Verbbegleiter durch bestimmte vom Verb determinierte syntaktische und semantische Eigenschaften auszeichnen, die notwendigerweise miteinander korrelieren, ist jedoch nicht unproblematisch. Die nachfolgenden Beispiele zeigen, dass ,es eine nicht unbeträchtliche Anzahl von Verben gibt, die ihre Begleiter nur in Bezug auf einige der von Tesnière identifizierten valenzrelevanten Eigenschaften definieren“ (Blume 2000: 9):

(37) a. Paula wohnt *(in der Stadt). (TvdB)

b. Johanna fährt diese Strecke ungern. (nach Blume 2000: 11)

c. Hans sucht ein Buch. vs.

Hans sucht nach einem Buch. (TvdB)

d. Kurt unterstützt seinen Schwager. vs.

Karl hilft seinem Schwager. (nach Blume 2000: 11)

Das Verb wohnen in (37a) in der Bedeutung jemand hat irgendwo seine Wohnung und lebt dort ständig (vgl. E-VALBU_wohnen; 03.06.2018) impliziert zwei Verbbegleiter: Einerseits eine Personenbezeichnung, andererseits eine (statische) Ortsangabe ${ }^{45}$. Wohnen öffnet somit zwei Leerstellen, deren syntaktische Realisierung - das zeigt der bei Elision der Verbbegleiter entstehende ungramma-

45 Das Verb wohnen in der hier betrachteten Lesart lässt alternativ zu einer (statischen) Ortsangabe auch eine Angabe zur Art und Weise zu (z. B. Paula wohnt ruhig.) 
tische Satz - obligatorisch ist. Obwohl somit beide Verbbegleiter eindeutige Aktanten-Eigenschaften zeigen, gilt bei Tesnière die Ortsangabe in der Stadt nicht als valenzgefordert. Sie ist weder als nominales Element realisiert noch weist sie eine der für Aktanten obligatorischen semantischen Rollen auf. Entsprechend ist die Lokalbestimmung im Sinne Tesnières nicht als valenzgesteuerter Aktant, sondern vielmehr als valenzunabhängige Angabe zu analysieren. Diese Annahme ist nicht unproblematisch, weil Angaben nach Tesnière ihrem Wesen nach fakultativ sind (vgl. Tesnière 1980: 116) - eine Auffassung, die aber gerade auf die statische Lokalbestimmung in dem Satz Paula wohnt in der Stadt nicht zutrifft.

Beispiele wie diese zeigen die Willkürlichkeit der Tesnièreschen Setzung, nach der ausschließlich nominale Elemente valenzgefordert Aktanten, Adverbien hingegen grundsätzlich valenzunabhängige Angaben darstellen (vgl. Engel 2006: 1316). Ein Umstand, der auch durch das nachfolgende Beispiel verdeutlicht wird: Das Verb fahren in (37b) in der Bedeutung jemand/etwas bewegt sich mit Hilfe einer Antriebskraft irgendwohin (vgl. E-VALBU_fahren; 03.06.2018) impliziert zwei Verbbegleiter: Einerseits eine Person bzw. ein Fahrzeug, andererseits eine (direktive) Ortsangabe. Der zweite Verbbegleiter von fahren bezeichnet also eine im Sinne Tesnières nichtvalenzgeforderte Umstandsangabe, die seiner Auffassung nach grundsätzlich als Adverbial zu realisieren ist. In dem in (37b) genannten Beispiel wird die Ortsangabe jedoch als nominales Element (diese Strecke) realisiert; sie müsste entsprechend als Aktant ${ }^{46}$ klassifiziert werden. Tesnière, der diesen Widerspruch bemerkt, gibt hierzu lediglich an, „daß solche Begleiter in einigen Sprachen als Aktanten empfunden werden“ (Blume 2000: 11).

Als ebenfalls problematisch erweist sich Tesnières Grundannahme, wonach die Kasus der einzelnen Aktanten stets mit bestimmten semantischen Rollen übereinstimmen (vgl. Tesnière 1980: 100ff.). Dass diese Annahme nicht immer zutrifft, zeigt u.a. das Beispiel (37c): Das Verb suchen in der Bedeutung jemand/etwas sieht (irgendwo) nach etwas Verlorenem (vgl. E-VALBU_suchen; 03.06.2018) lässt einerseits eine Realsierung seines Verbbegleiters als (im Sinne Tesnières valenzgeforderte) Akkusativ-NP zu; andererseits ist - bei gleichbleibender semantischer Rolle - aber auch eine Realisierung des Verbbegleiters als (nach Tesnière valenzunabhängige) Präpositionalphrase möglich. Ähnlich verhält es sich bei den Satzpaaren in (37d): Während das Verb unterstützen in der Bedeutung jemand/etwas hilft jemandem (vgl. E-VALBU_unterstützen; 03.06.2018) einen ersten Aktanten (Handelnder) und einen zweiten Aktanten (Betroffener) im Sinne Tesnières realisiert, weist das synonyme Verb helfen in der Bedeutung jemand/etwas ermöglicht

46 Eine Klassifizierung, für die sich auch Blume ausspricht, da „lokale Bestimmungen dieser Art selbst bei Bewegungsverben nicht frei hinzufügbar sind“" (Blume 2000: 11). 
jemandem/etwas, bei etwas erfolgreich zu sein (vgl. E-VALBU_helfen; 03.06.2018) einen ersten Aktanten (Handelnder) und einen dritten Aktanten (Nutznießer) auf. Und das, obwohl sich die Rolle des im Akkusativ realisierten Aktanten von unterstützen und die des im Dativ realisierten Aktanten von helfen intuitiv nicht unterscheiden (vgl. Blume 2000: 11f.).

Tesnière definiert Aktanten und Angaben „durch komplementäre Mengen von miteinander korrelierenden Eigenschaften“ (ebd. 12). Eine eindeutige Zuordnung der einzelnen Verbbegleiter ermöglichen diese Kriterien jedoch nicht - das haben die oben genannten Beispiele deutlich gemacht. Vielmehr lassen sich zahlreiche Verben ermitteln, die nur Teilmengen der genannten syntaktischen und semantischen Eigenschaften des einen oder des anderen Konzepts aufweisen, also strenggenommen weder als Aktanten noch als Angaben klassifiziert werden können (vgl. ebd.).

\subsection{Valenz als einheitliches Phänomen}

Auch Tesnière räumt gewisse Abgrenzungsprobleme zwischen Ergänzungen und Angaben ein, indem er schreibt:

Auf den ersten Blick ist die Abgrenzung zwischen Aktanten und Angaben eindeutig. Bei näherer Betrachtung stößt man freilich auf Schwierigkeiten, wenn man sie präzise durchführen will. Der [...] dritte Aktant [...] weist bereits einige Merkmale der Angaben auf. Umgekehrt weisen gewisse Angaben einige Analogien zu den Aktanten auf. (Tesnière 1980: 115)

In der Forschungsdiskussion werden zahlreiche Versuche unternommen, diesen Differenzierungsschwierigkeiten $\mathrm{zu}$ begegnen. Lange wird Valenz als (weitestgehend) einheitliches Phänomen - nämlich als die „Ergänzungsbedürftigkeit“ von Verben (und später auch anderer Wortarten) - betrachtet. Als Konsequenz dieses Valenzbegriffs wird der Unterscheidung zwischen valenzgeforderten Ergänzungen einerseits und nicht-valenzgeforderten Angaben ${ }^{47}$ andererseits ein zentraler Stellenwert zur Entwicklung einer adäquaten Valenztheorie beigemessen (vgl. Welke 2011).

So unverzichtbar die Abgrenzung zwischen Ergänzungen und Angaben scheint, so strittig ist sie auch (vgl. Helbig 1996: 45). So wird in der Folgezeit Tesnières Valenz-

47 Für Ergänzungen und Angaben sind im Laufe der Zeit ganz unterschiedliche Begriffe in der Forschungsliteratur verwendet worden: So finden sich etwa für Ergänzungen auch die Bezeichnungen Aktanten, Valenzpartner, Komplemente, Partizipanten oder Mitspieler, während für Angaben auch die Begriffe Zirkumstanten, (freie) Angaben, Umstandsbestimmungen, Supplemente, Modifizierer und Adjunkte gebräuchlich sind. Für einen Überblick über die terminologischen Varianten vgl. Storrer (2003). 
bindungsbeziehung von Wissenschaftlern keineswegs einheitlich gedeutet. Vielmehr stehen sich zumeist monokriterial ausgerichtete syntaktische Ansätze - vgl. etwa den auf Rektion und Nicht-Weglassbarkeit basierenden Valenzbegriff bei Eisenberg $\left(2013^{4}\right)$ - und semantische Konzeptionen - z. B. das im wesentlichen semantisch ausgerichtete Valenzkonzept der Projektgruppe Verbvalenz (1981) - gegenüber, in denen immer neue und bessere formale Kriterien zur Operationalisierung der intuitiv einleuchtenden Unterscheidung zwischen Ergänzungen und Angaben entwickelt werden (vgl. zusammenfassend Storrer 2003). Es gilt stets die Forderung, durch die jeweiligen Abgrenzungskriterien Ergänzungen und Angaben ausnahmslos richtig zu kategorisieren - ein Postulat, das aufgrund der eindimensionalen Ausrichtung der meisten Ansätze unmöglich einzulösen ist. So bleiben in allen Konzeptionen, die einem Verbbegleiter die Eigenschaft valenzgefordert aufgrund genau einer Eigenschaft zuweisen wollen, wichtige andere Valenzinformationen unberücksichtigt.

Der Forschungsdiskussion, die lange Zeit von der Suche nach der richtigen Valenzebene bzw. dem richtigen Abgrenzungskriterium für Ergänzungen und Angaben bestimmt ist (vgl. Storrer 2003: 778), gibt Jacobs (1994a, b) eine überraschende Wende (vgl. Ágel/Fischer 2010: 268). Für ihn ist Valenz nicht länger ein einheitliches Phänomen, sondern eine Art Oberbegriff für eine Reihe von voneinander unabhängigen extensionsverschiedenen Valenzbindungsbeziehungen.

\subsection{Valenz als multidimensionales Phänomen}

Die sich langsam durchsetzende Ansicht, „dass die Suche nach der einzigen und richtigen Grenzlinie zwischen Ergänzungen und Angaben ein müßiges Unterfangen ist, war [...] ein Befreiungsschlag für die Valenzforschung“ (Storrer 2003: 778), aus dem heraus sich eine multidimensionale Valenzkonzeption entwickelt ${ }^{48}$. In einem solchen Konzept, „wird Valenz als Bündelung von verschiedenen, voneinander unabhängigen Relationen formaler und semantischer Art verstanden“ (Hoberg 2006: 862). Es gilt damit für Viele ${ }^{49}$ als „Lösung der leidigen

48 Trotz des unter den meisten Valenzforschern bestehenden Konsenses, dass sich Valenz über verschiedene Sprachebenen ausdehnt, merkt Eroms (2001: 11) an, dass sich eine „bei allen Valenzgrammatikern vollkommen identische Auffassung, was Valenz ist, [...] bisher nicht [hat, TvdB] erreichen lassen“.

49 Die Idee eines mehrdimensionalen Valenzkonzepts ist nicht unumstritten. Kritiker bezweifeln die Zweckmäßigkeit der Abgrenzung von Valenzebenen. Entsprechend plädiert etwa Simmel (1994: 4) dafür, die Valenzebenen durch Rückbesinnung auf den Aufbau sprachlicher Zeichen aus Ausdrucks- und Inhaltsseite, eine Systematisierung der Einflüsse von Kontext und Situation sowie die Begründung eines systematischen Ersparungsbegriffs zu ersetzen. Auch Fischer $(2001,2003)$ zweifelt an der Sinnhaftigkeit eines multidimensionalen Valenzbegriffs. Er 
Klassifikationsprobleme der traditionellen Valenztheorie“ (Jacobs 1992a: 95), kann man nunmehr Verbbegleiter „sehr differenziert nach ihrer Bewertung in den einzelnen Valenzdimensionen ordnen und unterscheiden“ (ebd. 96).

„In ihrer expliziten Fassung geht die Idee eines multidimensionalen Valenzkonzepts auf Jacobs zurück“ (Zifonun et al. 1997: 1030). 1987 in Kontra Valenz erstmals vorgeschlagen, wird Jacobs' Valenzmodell seitdem kontinuierlich überarbeitet und zu einer multidimensionalen Valenztheorie ausgebaut. In der deutschen Valenzforschung breit diskutiert (vgl. Ágel (2000), Welke (2011)) und von anderen Forschern im Kern übernommen (z. B. Zifonun et al. (1997); Hölzner $(2007))^{50}$ ist sie auch für die folgenden Überlegungen von besonderer Bedeutung und soll daher kurz vorgestellt werden:

\subsubsection{Kategoriale und semantische Valenz}

Valenz bezeichnet die Eigenschaft von (verbalen) Ausdrücken, die Satzumgebung, in der sie vorkommen, in einer bestimmten Art und Weise vorzustrukturieren. So kann z. B. eine Verbform wie versteckt in die Sätze in (38), nicht jedoch in die in (39) eingesetzt werden:
a. Peter den Schlüssel.
b. Peter sich.
c. Peter den Schlüssel unter der Bank.
a. Peter dem Schlüssel.
b. Der Stein Peter.
c. Peter

Viele der in (38) und (39) sichtbaren Umgebungsrestriktionen für die Verbform versteckt lassen sich erfassen, wenn man davon ausgeht, dass versteckt zwei Ergänzungen - eine im Nominativ und eine im Akkusativ - fordert. Hieraus folgt, dass das Verb nicht in die Sätze (39a) - mit realisiertem Dativobjekt - und (39c) ohne Realisierung einer zweiten Ergänzung - eingesetzt werden kann. Notiert

schlägt stattdessen ein unidimensionales Valenzmodell mit Sachverhaltskonstitution, d. h. dem Entwurf einer Aussage, als einzigem (partiell indeterminierten) Kriterium vor.

50 Weitere Arbeiten, die Ergebnisse des von Jacobs entwickelten multidimensionalen Valenzkonzepts aufgreifen, sind u. a. Breindl (1989), Butulussi (1991), Storrer (1992) und Primus (1999). Kritische Anmerkungen zum multidimensionalen Valenzkonzept finden sich z. B. in Zifonun (1995) sowie Fischer (1999) und (2003). 
wird eine entsprechende Forderung nach Jacobs als ,/nom/akk‘. Aufgebaut aus den kategorialen Merkmalen Nominativ und Akkusativ ordnet Jacobs ,/nom/akk den kategorialen Merkmalen von versteckt zu. Eine solche Einbeziehung kategorialer Valenzmerkmale in die syntaktische Kategorie des jeweiligen Valenzträgers ist nach Jacobs (1992a) unproblematisch, da sie sich hinsichtlich relevanter Generalisierungen - vgl. z. B. die Unterwerfung unter das für kategoriale Merkmale typische Hauptprinzip (vgl. ebd.) - nicht von anderen kategorialen Merkmalen unterscheiden. In diesem Sinne entspricht die syntaktische Kategorie von versteckt der Darstellung in (40), wobei die den syntaktischen Valenzangaben vorangehenden Merkmale die Wortart des Ausdrucks, seine morphosyntaktischen Eigenschaften sowie eventuell weitere Merkmale (angezeigt durch den Platzhalter [...]) charakterisieren:

(40) $\{\mathrm{V}$, ind, präs, sing, 3pers, [...], / $\{\mathrm{N}$, nom $\} /\{\mathrm{N}$, akk $\}\}$

Zwar lassen sich mit Hilfe der kategorialen Valenzforderung einige der in (38) und (39) dargestellten Umgebungsbeschränkungen erklären; darüber hinaus bedarf es jedoch zusätzlicher Annahmen, um etwa die Unmöglichkeit von Strukturen wie in (39b) zu erfassen. Das Problem besteht hier darin, dass Entitäten, die etwas verstecken, belebt sein müssen; eine Forderung, die auf Steine gerade nicht zutrifft. Um derartige Restriktionen zu erfassen, greift Jacobs auf die sog. semantische Repräsentation $(\mathrm{SR})^{51}$ eines Valenzträgers zurück. Dem Verb verstecken entspricht z. B. die semantische Repräsentation VERSTECK [s] (x) (y). Das bedeutet, dass das Verb verstecken ein dreistelliges Prädikat ist, das sich auf Situationen (angezeigt durch die Relatvariable [s]) bezieht, in denen ein Individuum zu einem anderen in eine bestimmte Beziehung - nämlich die verstecken-Beziehung - tritt (angezeigt durch die Relatvariablen (x) und (y)). Bei Äußerungen, in denen das Verb benutzt wird, werden die Relatvariablen mit passenden Entitäten belegt. Die damit einhergehenden Restriktionen, die z. B. die Unmöglichkeit von (39b) erfassen - verstecken setzt die zwingende Belebtheit des versteckenden Individuums voraus, ein Umstand, der nun gerade auf Steine nicht zutrifft -, können mit Hilfe von sog. Bedeutungsregeln (BR), die sich auf die semantische Repräsentation des Verbs beziehen, formuliert werden, vgl. (41a). Durch entsprechende Subskripte

51 Die semantische Repräsentation bringt unabhängig von der Valenz zum Ausdruck, „dass man mit dem Verb über Situationen spricht, in denen ein Individuum zu einem anderen in eine bestimmte Beziehung tritt“ (Jacobs 2003: 380). 
kann in den semantischen Repräsentationen auf die Bedeutungsregeln hingewiesen werden, vgl. (41b) $)^{52}$ (vgl. Jacobs $1994 b$ und 2003):
a. BR: $\forall \mathrm{x} \forall \mathrm{y} \forall \mathrm{s}$ [VERSTECK [s](x)(y) $\rightarrow$ KONKRET(x) \& BELEBT(y) \& ENDLICH [s]]

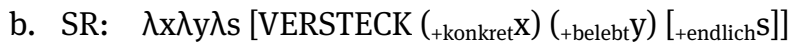

Die Gesamtheit der Relatpositionen bezeichnet Jacobs als semantische Valenz eines Valenzträgers. Er geht davon aus, dass die semantischen Valenzmerkmale einen Teil der semantischen Kategorie des jeweiligen Valenzträgers bilden. Die semantische Kategorie von versteckt lässt sich dementsprechend wie in (42) darstellen:

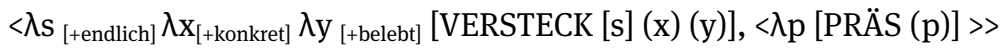

Die auf die semantischen Valenzangaben in (42) folgende, hier eingliedrige

Folge von Funktoren charakterisiert funktionale Bedeutungsbestandteile des Ausdrucks (seine ,funktionale Valenz'), z. B. die Tempusbedeutung, die sich beim Prozeß der Bedeutungskomposition anders verhalten als die semantische Valenz.

(Jacobs 1992a: 99)

Valenz, das haben die vorangehenden Darstellungen gezeigt, bezeichnet also die Fähigkeit eines Lexems, seiner syntaktischen und seiner semantischen Umgebung Beschränkungen aufzuerlegen. Entsprechend muss Valenz also sowohl auf kategorialer (d.h. syntaktischer) als auch auf semantischer Repräsentationsebene ${ }^{53}$ des Lexikons angesiedelt werden. Beschränkungen auf nur eine Ebene, das haben die monokriterial ausgerichteten Ansätze der Vergangenheit gezeigt, würden wesentliche Teile der Kombinationsrestriktionen von Verben nicht erfassen.

Zur Präzisierung dieser Ebenendifferenzierung ${ }^{54}$ führt Jacobs weitere, auf beiden Valenzebenen beobachtbare Teilphänomene an, sog. Valenzdimensionen $^{55}$. Ausgehend von der in Jacobs (2003) verwendeten Terminologie, die im

52 Hier und in den nachfolgenden Darstellungen entspricht die Anordnung der Argumentvariablen der Reihenfolge ihrer Sättigung. Damit ergibt sich eine Sortierung vom rangniedrigsten (i.d.R. dem direkten Objekt) bis hin zum ranghöchsten Argument (dem Subjekt des Satzes).

53 Neben semantischen und syntaktischen Aspekten nehmen einige Forscher auch eine diskurspragmatische Dimension an. Eine ausführliche Darstellung hierzu findet sich bei Majorin (2008). 54 Für eine ausführliche Herleitung der Ebenendifferenzierung in kategoriale und semantische Valenz vgl. Jacobs (2003).

55 Jacobs listet ursprünglich sieben Valenzdimensionen. Die Dimensionen „Exozentrizität“ und „Assoziiertheit“ sowie die Unterdimensionen der „Formspezifik“, „DEFOSP“, und der „Inhaltsspezifik“, „DEINSP“, wurden später entfernt; sie erwiesen sich nicht als eigenständige, sondern 
Unterschied zu den zuvor verwendeten Begrifflichkeiten Notwendigkeit, formale Spezifität, inhaltliche Spezifität, Argumenthaftigkeit und Beteiligtheit (vgl. Jacobs 1994a) eine stärkere Fokussierung auf die vom Verb als Valenzträger ausgehenden Forderungen an seine jeweiligen Begleiter sowie die prinzipielle Unabhängigkeit und Gleichgewichtigkeit der einzelnen Valenzdimensionen deutlicher abzubilden scheint, handelt es sich hierbei im Einzelnen um:

(43) Kategoriale (syntaktische) Valenzdimensionen:

Realisierungsforderungen $\quad(\approx$ Notwendigkeit $)$

Merkmalsforderungen $\quad(\approx$ formale Spezifität $)$

(44) Semantische Valenzdimensionen:

Relatforderungen ( $\approx$ Argumenthaftigkeit)

Sortale Forderungen $\quad(\approx$ inhaltliche Spezifität $)$

Rollenforderungen $\quad(\approx$ Beteiligtheit/Partizipation)

Im Weiteren werden zunächst die einzelnen Valenzdimensionen, auf denen die wortspezifischen Informationen über mögliche Satzumgebungen eines Valenzträgers expliziert werden, am Beispiel des Verbs verstecken überblicksartig dargestellt. Um Verbstellungsproblematiken auszublenden, werden hierbei nur Nebensatzstrukturen betrachtet. Da syntaktische und semantische Valenzdimensionen jeweils eigenen Gesetzmäßigkeiten folgen (vgl. Jacobs 1992a), werden syntaktische und semantische Forderungen getrennt voneinander repräsentiert. Dieses Repräsentationsformat von Valenz wird auch der Beobachtung gerecht, dass alle Valenzdimensionen - trotz einzelner (unilateraler) Implikationsverhältnisse (vgl. hierzu ausführlich Jacobs 1994a) - autonom sind in dem Sinne, dass nicht zwei Valenzbindungsbeziehungen notwendigerweise immer zusammen auftreten.

\subsubsection{Dimensionen von Valenz}

\subsubsection{Kategoriale Dimensionen}

Als kategoriale Valenz (im Folgenden auch syntaktische Valenz) werden die morphosyntaktischen Valenzmerkmale einer Wortform bezeichnet (vgl. Jacobs 2003). Für ein Verb wie verstecken wird die syntaktische Valenz durch ,/nom/akk notiert. In dieser Notation sind zwei verschiedene Arten von Forderungen an die

als in die übrigen Valenzdimensionen integrierte Phänomene (vgl. Nachschrift zu „Kontra Valenz“ (Jacobs 1994a)). 
jeweilige syntaktische Umgebung eines verbalen Valenzträgers enthalten: sog. Realisierungs- und Merkmalsforderungen.

\section{Realisierungsforderungen}

Die Repräsentation der kategorialen Valenz (auch KR-Valenz) enthält zunächst Informationen über die Anzahl der Valenzstellen, die ein Verb öffnet. Sie werden durch einen Schrägstrich (/) markiert. Für das Verb verstecken mit der syntaktischen Valenz ,/nom/akk' ergibt sich demnach die Forderung nach Realisierung von zwei syntaktischen Begleitern (vgl. (45a)). Werden dagegen zu wenige Verbdependentien realisiert, führt das, wie in (45b), zur Ungrammatikalität des Satzes:

(45) a. (weil) Peter den Schlüssel versteckt. (TvdB)

b. ${ }^{\star}$ (weil) Peter versteckt. (ebf.)

Die Unmöglichkeit von Strukturen wie (45b) erfasst Jacobs über die von ihm explizierte Bedingung der syntaktischen Verarbeitung von kategorialen Valenzmerkmalen (s. Jacobs 1992a, 1992b). Hiernach gilt, dass jede

der im KR-Valenzmerkmal $/ \mathrm{m}_{1} / \ldots / \mathrm{m}_{\mathrm{n}}$ einer valenztragenden Konstituente VT enthaltene Forderung $/ \mathrm{m}_{\mathrm{i}}[$...] entweder a) durch eine Schwesterkonstituente von VT erfüllt und dann gelöscht oder b) zur Mutterkonstituente transferiert werden [muss, TvdB] (unter Beachtung des Hauptprinzips).

(Jacobs 2003: 382)

Diese Bedingung wird in (45b) offensichtlich verletzt, da die Realisierung lediglich eines syntaktischen Verbbegleiters nicht ausreicht, um die vom Verb verstecken ausgehenden Realisierungsforderungen für zwei Begleiter zu sättigen.

Unproblematisch sind dagegen Strukturen wie in (46), worin die von der oben genannten Bedingung vorausgesetzte „Möglichkeit des Transfers von KRValenzforderungen zu einer Projektion des Valenzträgers“ (ebd. 383) dargestellt ist:

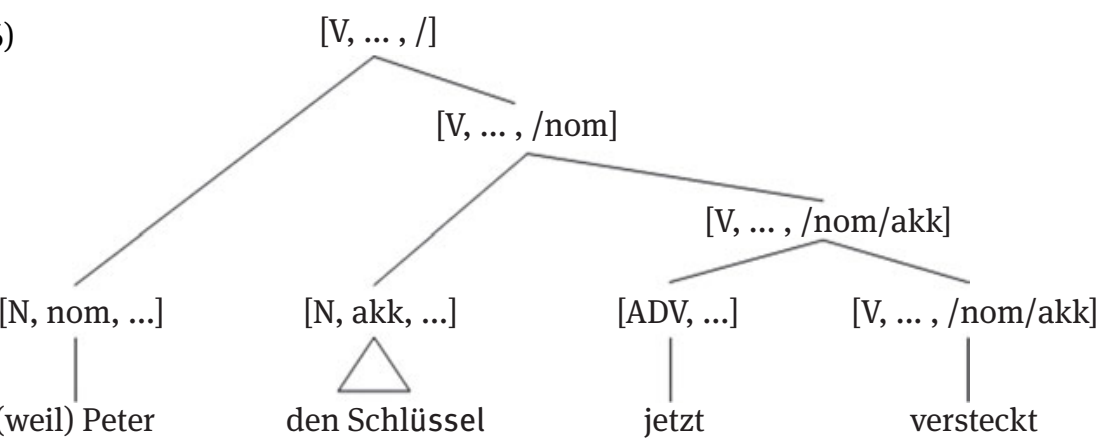


In Beispielsatz (46) übernimmt zunächst die Mutterkonstituente - unter Beachtung des Hauptprinzips - die syntaktische Valenz ihrer Tochterkonstituente. Anschließend findet eine schrittweise Stellensättigung durch die Schwesterkonstituenten statt, wobei alle einschlägigen Gesetze berücksichtigt werden.

Problematischer verhält es sich dagegen mit Sätzen wie in (47); sie werden durch die mit der oben genannten Bedingung verbundenen Möglichkeit zur Klärung der „für die Realisierung [der kategorialen Valenzmerkmale, TvdB] in Frage kommende strukturelle Position“ (ebd. 382) ausgeschlossen:

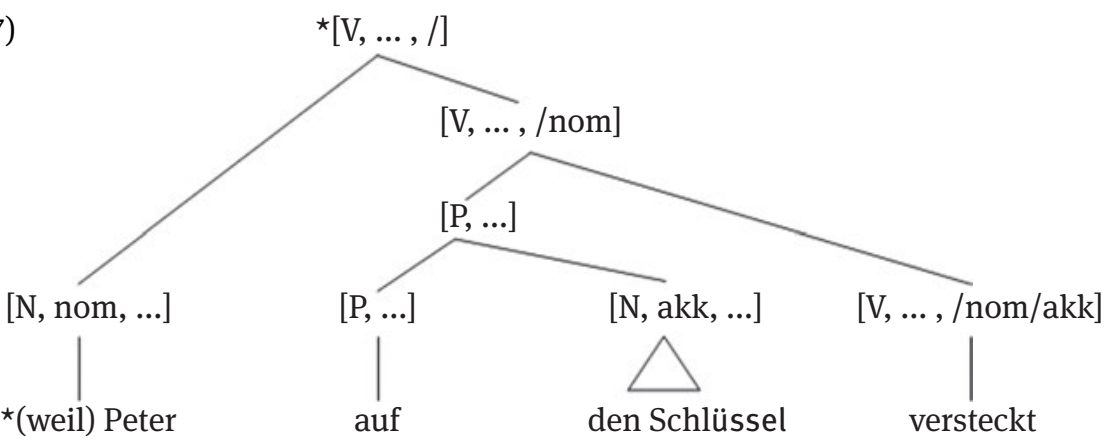

In (47) wird zwar - anders als in (45b) - ein Akkusativ-Nominal realisiert; da es sich hierbei jedoch nicht um ein Dependens des Verbs handelt, ist es für dessen ,/akk'-Forderung nicht greifbar - sie bleibt entsprechend ungesättigt.

Überprüft werden können die (syntaktischen) Realisierungsforderungen eines Valenzträgers u. a. durch Weglassung der jeweiligen Konstituenten, die die entsprechenden Valenzstellen füllen ${ }^{56}$. So stehen das Verb und seine die Realisierungsforderungen des Valenzträgers erfüllenden Begleiter in einer syntagmatischen Beziehung der Notwendigkeit zueinander (vgl. ebd. 390). Dieses Verfahren erinnert an die oft kritisierte Weglassprobe (etwa bei Helbig/Schenkel 19837), deren Ergebnisse in der einschlägigen Literatur häufig zu widersprüchlichen Beurteilungen hinsichtlich der Notwendigkeit eines Verbbegleiters führten ${ }^{57}$. Blume (1993) konnte jedoch zeigen, dass die mit der Weglassprobe verbundenen Probleme durch restriktive Anweisungen bei der Konstruktion der Testsätze kontrolliert werden können. In diesem Sinne werden im Rahmen dieser Arbeit die von

56 Weitere Testverfahren, mit denen obligatorische Ergänzungen in relativ-obligatorische und absolut-obligatorische Ergänzungen weiter subklassifiziert werden können, wurden u. a. von Pasch (1977: 23ff) vorgeschlagen.

57 Zur Kritik der Weglassungsprobe vgl. u.a. Nikula (1978), Welke (1988) und Storrer (1992) sowie (2003). 
Verben ausgehenden Realisierungsforderungen mit Hilfe eines standardisierten Eliminierungstests ${ }^{58}$ (s. Kapitel 1.3) überprüft.

\section{Merkmalsforderungen}

Neben Realisierungsforderungen sind mit den meisten syntaktischen Valenzstellen eines Verbs auch formale Merkmalsforderungen für die zu realisierenden syntaktischen Begleiter verbunden. Entsprechend müssen z. B. die Dependentien des Verbs verstecken - das geht aus der syntaktischen Valenz ,/nom/akk hervor - die Merkmale Nominativ und Akkusativ aufweisen. Die Verwendung anderer Strukturen führt dagegen zur Ungrammatikalität des Satzes, vgl. (48):

(48) a. (weil) Peter den Schlüssel versteckt.

b. *(weil) Peter dem Schlüssel versteckt.

Um Strukturen wie (48b) tatsächlich auszuschließen, formuliert Jacobs eine Zusatzbedingung für die oben genannte Bestimmung zur syntaktischen Verarbeitung von KR-Valenzmerkmalen. Hierin heißt es:

Eine Konstituente X erfüllt nur dann die in der Stelle $/ \mathrm{m}_{\mathrm{i}}$ des Merkmals $/ \mathrm{m}_{1} / \ldots / \mathrm{m}_{\mathrm{n}}$ enthaltene Forderung, wenn $\mathrm{m}_{\mathrm{i}}$ in der Kategorie von $\mathrm{X}$ enthalten ist.

(Jacobs 2003: 383)

Von den syntaktischen Merkmalsforderungen des Valenzträgers erfasst wird u. a. der Kasus eines Objekts - das zeigen die Beispiele in (48b) und (50g). Darüber hinaus können auch Adpositionen, Infinitivkonstruktionen und satzwertige Objekte valenzgefordert sein, vgl. (49); hierbei sind jedoch u. U. auch sortale Forderungen in Erwägung zu ziehen (vgl. ebd. 384).

(49) a. (weil) Peter auf Susi wartet. (TvdB)

b. (weil) Peter nicht zu fragen braucht. (ebf.)

c. (weil) Peter fragen will. (ebf.)

d. (weil) Peter glaubt, dass Susi morgen kommt. (ebf.)

58 Der Eliminierungstest darf nicht mit der von Grebe eingeführten Abstrichprobe (vgl. Grebe 1959) verwechselt werden. Auch hier wird zwar ein Valenzkandidat des jeweiligen Valenzträgers eliminiert (,abgestrichen“), „die Bewertung des Restsatzes erfolgt aber im Hinblick auf das semantische Kriterium der Sinnnotwendigkeit“ (Storrer 2003: 772), das in etwa der vom Valenzträger ausgehenden Relatforderung entspricht. 
Doch nicht alle im Satz auftretenden Merkmale stehen unter dem Einfluss der Valenz:

(50) a. (weil) Peter den Schlüssel versteckt hat.

b. (weil) Peter den Schlüssel, den er gestern gefunden hat, versteckt hat.

c. (weil) Peter einen Schlüssel versteckt hat.

d. (weil) Peter ihn versteckt hat.

e. (weil) Peter die Schlüssel versteckt hat.

f. (weil) Peter seinen Schlüssel versteckt hat.

g. *(weil) Peter dem Schlüssel versteckt hat.

Die Beispiele in (50) machen deutlich, dass die interne Struktur der syntaktischen Begleiter eines Valenzträgers nicht von dessen kategorialer Valenz abhängt: So ist es für die Valenz von verstecken unerheblich, aus wie vielen Wörtern das direkte Objekt besteht (50a,b), ob es definit oder indefinit (50a,c), pronominal oder nicht pronominal (50a,d) auftritt. Ebenfalls von der KR-Valenz von verstecken unabhängig sind Personen-, Genus- und Numerusmerkmale, auch wenn sie wie in (50f) von anderen Konstituenten beeinflusst werden. Sie werden - mit Blick auf die strukturellen Bedingungen der Sättigung von KR-Valenzmerkmalen (s. o.) - i.d.R. über verschiedene Formen der Kongruenz mit der entsprechenden Konstituente festgelegt (vgl. ebd. 384f.).

Um zu überprüfen, ob eine Konstituente Merkmalsforderungen ${ }^{59}$ des verbalen Valenzträgers unterliegt, kann u. a. die sog. Austauschprobe ${ }^{60}$ durchgeführt werden. So können Konstituenten, die formalen Merkmalsforderungen unterliegen, aufgrund ihrer syntagmatischen Beziehung der Formregiertheit zum Verb (vgl. ebd. 390) nicht durch andere Merkmale ausgetauscht werden, ohne dass der daraus resultierende Ausdruck ungrammatisch wird. Die Austauschprobe führt allerdings - ähnlich wie die oben genannte Weglassprobe - $\mathrm{zu}$ falschen Ergebnissen, wird sie nicht in weitergehende theoretische Überlegungen (z. B. in die

59 Für Jacobs liegen formale Merkmalsforderungen vor, wenn die Form möglicher Valenzkandidaten vom jeweiligen Valenzträger aufgrund spezifischer Forderungen festgelegt wird. Zifonun et al. (1997: 1034ff.) differenzieren dabei feiner in die drei rektionalen Formrelationen Rektion bezeichnet die Kasusfestlegung bzw. die Festlegung anderer Rektionskategorien durch den Valenzträger -, Konstanz - bezeichnet die Festlegung einer Präposition durch den Valenzträger - und Kasustransfer - bezeichnet die zusätzliche Kasusfestlegung der von der Präposition regierten Kasus durch den Valenzträger.

60 Die sog. Austauschprobe wird auch als Substitutions- oder Subkategorisierungstest bezeichnet. 
Überprüfung von Kongruenzverhältnissen) eingebettet. So kann man bei naiver Anwendung des Tests

zwar erkennen, ob die Realisierung bestimmter Merkmale in einer Satzposition X von der Besetzung einer anderen Position Y abhängt, aber sie lassen [...] die Gründe für solche Abhängigkeiten unklar, können also z. B. nicht unterscheiden, ob X durch die Valenz von $Y$ oder über Kongruenz mit $\mathrm{Y}$ beeinflusst wird.

(ebd. 385)

\section{Repräsentationsformat kategorialer Valenzforderungen}

Auf Grundlage der obigen Beschreibungen ergibt sich für die syntaktische Valenz (im Folgenden auch SynVal) in Anlehnung an die von Jacobs (1994b) vorgeschlagene Valenzdarstellung das in (51) für das Verb verstecken exemplarisch aufgeführte, für weitere Überlegungen zentrale Repräsentationsformat:

(51) SynVal: $/$ nom $^{\mathrm{y}} / \mathrm{akk}^{\mathrm{x}}$

In dieser Repräsentation enthalten sind zunächst - durch Schrägstrich markiert - Informationen über die Anzahl der syntaktischen Begleiter, die ein Verb fordert, für verstecken zwei. Darüber hinaus enthält die Notation die mit den syntaktischen Valenzstellen verbundenen formalen Merkmale - bei dem Verb verstecken die Kasus Nominativ und Akkusativ.

Die in (51) zusätzlich vorgenommene Indizierung der KR-Valenz macht die nachweisbare Korrespondenz zwischen der syntaktischen und semantischen Valenzebene deutlich. Sie wird in Kapitel 2.3.2.2 noch deutlicher herausgearbeitet.

Im weiteren Verlauf spielen syntaktische Valenzangaben neben der reinen Darstellung verbaler Valenzträger auch bei der im Sinne von F2 durchzuführenden Lesartendifferenzierung eine zentrale Rolle (vgl. hierzu auch Kapitel 3.2). So ist schon oft beobachtet worden, dass ,die Argumentstruktur vieler Verben variieren [kann, TvdB] und nicht selten [...] Änderungen in der Syntax mit semantischen Differenzen [korrelieren, TvdB]“" (Willems/Coene 2003: 38), vgl. exemplarisch das Verb schreien in (52) und (53):

(52) schreien

i.S.v. jemand gibt unartikulierte Laute großer Lautstärke von sich (vgl. E-VALBU_schreien; 29.08.2018)

Bsp.: Die Kinder schreien. (TvdB)

SynVal: /nom 
schreien

i.S.v. jemand/etwas verlangt mit lauter, schriller Stimme, dass jemand kommt, etwas gebracht wird, oder dass man etwas bekommt (vgl. E-VALBU_schreien; 29.08.2018)

Bsp.: $\quad$ Renate schreit nach der Polizei. (TvdB)

SynVal: /nom /Prp ${ }^{\text {nach }}$

Daher werden morphosyntaktische Valenzmerkmale im Folgenden auch bei der Lesartenunterscheidung der hier zu untersuchenden Verben eingesetzt ${ }^{61}$. Welche Verfahren bei der Ermittlung entsprechender Merkmale im Einzelnen zur Anwendung kommen, wird in Kapitel 4 eingehend thematisiert.

\subsubsection{Semantische Dimensionen}

Die semantische Valenz von verstecken bildet nach Jacobs die Gesamtheit der Relatpositionen in der semantischen Repräsentation VERSTECK [+endlich $\mathrm{s}$ ( ${ }_{+ \text {konkret }} \mathrm{x}$ )

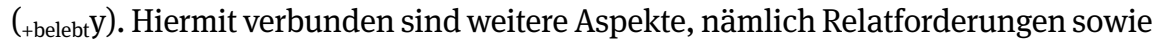
verschiedene Arten von inhaltlichen Forderungen an die einzelnen Relate (im Einzelnen: sortale Merkmalsforderungen und Rollenforderungen); sie werden im weiteren Verlauf dargestellt.

\section{Relatforderung}

Hinter dieser Valenzdimension verbirgt sich die Idee, dass bei jeder Äußerung eines verbalen Valenzträgers stets eine bestimmte Anzahl von Entitäten mitgedacht werden muss. Relatpositionen entsprechen somit den offenen Stellen in der Bedeutung eines Verbs (vgl. Engelberg 2000: 115).

61 Um der Proliferation valenzbedingter Lesarten entgegen zu wirken, werden immer wieder auch konstruktionsbasierte Modellierungen diskutiert (vgl. Proost 2015: 77). So nimmt Proost für das in (53) dargestellte Verb schreien in der Verwendung schreien nach eine eigene SUCH-Konstruktion ASM 14: nach etwas schreien - an (vgl. ebd. 62ff.), um eine drastische „Vermehrung konstruktionsspezifischer Lesarten [...], die in vielen Fällen intuitiv wenig plausibel erscheinen, wie z. B. [...] die Lesart ,fordern“ für schreien, brüllen oder krakeelen“ (ebd. 75f.) zu vermeiden.

Während sich eine Berücksichtigung konstruktionsbasierter Ansätze bei Verben, bei denen sich das gemeinsame Auftreten z. B. mit einer Präpositionalphrase ganz oder teilweise durch allgemeine syntaktische oder semantische Regeln herleiten lässt (vgl. Proost 2009: 12), als durchaus sinnvoll erweist, erscheint sie für die in den Kapiteln 5 und 6 dargestellten Verben wenig erklärungsstark. Die dort untersuchten Phänomene lassen sich vollständig über verb(lesarten)spezifische Valenzeigenschaften erfassen und erklären. Konstruktionsgrammatische Erklärungsansätze können dabei nicht mehr leisten als valenzbasierte Ansätze; sie bleiben daher nachfolgend unberücksichtigt. 
Wird beispielsweise das Verb verstecken verwendet, werden automatisch drei Entitäten mitgedacht: Zwei Individuen $\mathrm{x}$ und $\mathrm{y}$ und eine Situation s, in der die verstecken-Beziehung zwischen $\mathrm{x}$ und $\mathrm{y}$ besteht ${ }^{62}$. Hieraus ergibt sich insofern auch eine Forderung an die syntaktische Umgebung des Verbs, ,als in Sätzen, in denen das Verb vorkommt, Zahl und Art der Argumente des Verbs zu diesem Ensemble von Relatpositionen passen müssen“ (Jacobs 2003: 386). Als Argumente werden all jene syntaktischen Begleiter eines Verbs bezeichnet, die eine Relatposition in der semantischen Valenz des Verbs durch zusätzliche Informationen spezifizieren. In (54) stehen also Peter, den Schlüssel und jetzt in einer syntagmatischen Beziehung der Argumenthaftigkeit $\mathrm{zu}$ verstecken (vgl. ebd. 390), wobei das Dependens Peter besagt, dass das Verbrelat x eine Person namens Peter ist, den Schlüssel darüber informiert, dass das Verbrelat y ein Schlüssel ist und jetzt angibt, dass die vom Verb beschriebene Situation s umgehend eintritt:

(54) (weil) Peter den Schlüssel jetzt versteckt.

Dass Zahl und Art der Argumente eines Verbs zu dessen Relatforderungen passen müssen, bedeutet jedoch „nicht, dass es zu jeder Relatposition genau ein Argument im Satz geben muss“ (ebd.). Relatpositionen können in bestimmten Fällen auch unspezifiziert bleiben, etwa wenn „the verb has a variant with an explicit argument (i. e. an argument that gets syntactically realized) in the same semantic relation“ (Engelberg 2002: 375). In diesem Sinne weist die valenzreduzierte Variante des Verbs lesen in dem Satz in (55b) ein implizites Argument auf. In (55c) „kommt das dadurch zum Ausdruck, dass es kein das Relat y näher spezifizierendes Prädikat gibt“ (Jacobs 2003: 386) ${ }^{63}$ :

(55) a. (dass) Peter ein Buch liest (TvdB)

b. (dass) Peter liest

c. PETER $[\mathrm{x}]$ \& LIEST $[\mathrm{s}](\mathrm{x})(\mathrm{y})$

Bei entsprechenden Fällen handelt es sich um sog. fakultative Ergänzungen; sie sind Gegenstand nachfolgender Untersuchungen und werden daher in Kapitel 2.4 noch eingehend dargestellt.

62 Das Verfahren, die jeweils beschriebene Situation als Relat des Verbs aufzufassen, geht auf Davidson (1967) zurück. Eine ausführliche Begründung hierfür findet sich bei Engelberg (2000). 63 Der Frage, inwieweit die Annahme eines impliziten Arguments für intransitive Verwendungen von Verben wie lesen tatsächlich zutreffend ist, wird in Kapitel 6 nachgegangen. 
Implizite Argumente liegen auch dann vor, wenn „there is a morphologically related verb with an explicit argument in the same semantic relation" (Engelberg 2002: 375). Das obligatorisch intransitive Verb zuschlagen in (56), das morphologisch verwandt ist mit der transitiven Form schlagen, beschreibt z. B. eine Situation, in der ein Individuum einem anderen Individuum einen Schlag versetzt. Während der Schlagende durch ein Argument spezifiziert wird, bleibt der Geschlagene ungenannt - entsprechend fehlt auch in der Bedeutungskomposition in (56b) ein das Relat y näher kennzeichnendes Prädikat ${ }^{64}$ :

(56) a. (dass) Peter zuschlägt (nach Jacobs 2003: 386)

b. PETER $[\mathrm{x}]$ \& ZUSCHLÄGT $[\mathrm{s}](\mathrm{x})(\mathrm{y})$

Relatpositionen können jedoch nicht nur implizit bleiben; vielmehr können sie auch durch mehrere Argumente erfasst werden. In Konstruktionen mit prädikativen Adverbialen wird z. B. ein Relat durch mehrere Argumente spezifiziert, vgl. (57). Voraussetzung hierfür ist, dass sie nicht verschiedene valenzgeforderte Kasus aufweisen:

(57) a. (dass) Peter müde nach Hause kam. (ebd.)

b. PETER $[\mathrm{x}]$ \& MÜDE $[\mathrm{x}]$ \& NACH-HAUSE-KAM $[\mathrm{s}](\mathrm{x})$

Relatpositionen unterscheiden sich dadurch, ob sie referenziell oder nichtreferenziell sind: Während referenzielle Relatpositionen Entitäten beinhalten, „auf die man sich mit dem Valenzträger bezieht und die man durch ihn zumindest teilweise identifiziert“, werden die Entitäten in nicht-referenziellen Relatpositionen „erst durch hinzukommende Argumente des Valenzträgers identifiziert“ (ebd. 387). Während Argumente, die referenzielle Relate des Verbs spezifizieren, auch als Modifizierer bezeichnet werden, werden solche, die nicht-referenzielle Relate des Verbs spezifizieren, auch Identifizierer genannt. Mit Jacobs gehe ich davon aus, dass Situationsstellen als referenzielle Relatpositionen und Individuenrelate als nicht-referenzielle Relatpositionen zu betrachten sind. Eine entsprechende Unterscheidung ist für die nachfolgenden Überlegungen insofern bedeutsam, als sich die anschließenden Darstellungen und Diskussionen $\mathrm{zu}$ fakultativen

\footnotetext{
64 Die Möglichkeit von mitverstandenen, aber nicht-realisierten Relaten ist vom jeweiligen Valenzträger abhängig; sie muss entsprechend im Lexikoneintrag des Verbs festgehalten werden. Das intransitive Verb dinieren etwa „will not be represented as having two thematic arguments, since there is no morphologically related transitive verb. The fact that the involvement of some objects in the event - like the food with dine - is implied by the verb seems an insufficient reason to assume an implicit argument.“ (Engelberg 2002: 376).
} 
Ergänzungen nicht mit Modifizierern, sondern mit weglassbaren Identifizierern beschäftigen. Daher wird im Rahmen der im Anschluss vorgestellten Untersuchung zu den semantischen Konsequenzen bei Nicht-Spezifizierung einer Komplementstelle (Kapitel 5 und 6) bei der Darstellung der semantischen Valenzen auf die Nennung entsprechender Situationsvariablen verzichtet. Sie dienen lediglich der Verankerung nicht-valenzabhängiger Adverbiale, die im Zusammenhang mit den hier vorgenommenen Untersuchungen nicht von Bedeutung sind.

Ermittelt werden Relatpositionen i.d.R. mit Hilfe des sog. Folgerungs- oder Implikationstests: So folgt z. B. aus Peter isst einen Apfel $\rightarrow$ Es gibt eine Situation, die darin besteht, dass Peter einen Apfel isst. „Wie andere Diagnoseverfahren der Valenztheorie [...] führen jedoch auch Tests für Relatpositionen leicht in die Irre, wenn sie nicht durch theoriebezogene Überlegungen gestützt werden.“ (ebd. 386f.) Eine naive Anwendung des Implikationstests führt zu widersprüchlichen Ergebnissen etwa hinsichtlich der Argumenthaftigkeit von benefaktiven Dativen: Er trägt den Koffer $\neq>$ Es gibt jemanden, dem er den Koffer trägt. Hier weist das Verb tragen keine Relatposition für den Nutznießer der Handlung auf. Das Dativdependens wäre in Sätzen wie Er trägt ihr den Koffer somit kein Argument des Verbs, und das, obwohl es sich - wie Engelberg (2000) zeigen konnte - in anderen Zusammenhängen durchaus wie ein Argument verhält. Man kann benefaktive Dative z. B. keinem schon vorhandenen Dativ-Argument hinzufügen, vgl. ${ }^{*}$ Seiner Frau hilft er seinem Schwager (vgl. Jacobs 2003: 387). Jacobs umgeht dieses Problem, indem er entsprechende Strukturen als markierte Lesart und damit als eine Art Polysemie analysiert, für die ein zusätzlicher Lexikoneintrag erforderlich ist. Transitive Handlungsverben weisen demnach (mindestens) zwei Lesarten auf65: Eine mit und eine ohne durch einen Dativ zu spezifizierende Relatposition (vgl. ebd.).

Nach Ermittlung der verbspezifischen Relatpositionen können über den sog. geschehen-Test die referenziellen Relate - also Modifizierer - ausgefiltert werden. So ermöglicht die Anwendung des geschehen-Tests eine Unterscheidung zwischen Argumenten, die das Situationsrelat spezifizieren, und anderen Verbdependentien: Peter isst einen Apfel mit Messer und Gabel - Peter isst einen Apfel, und das geschieht mit Messer und Gabel (vgl. Engelberg 2000: 88ff.).

\section{Sortale Merkmalsforderung}

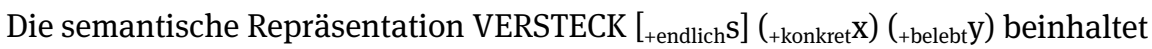
nicht nur die Forderung nach einer bestimmten Anzahl mitzudenkender Entitäten, sondern stellt auch den Anspruch, dass die entsprechenden Entitäten von

65 Dass die Annahme unterschiedlicher Lesarten nicht nur zur Erfassung benefaktiver Dative sinnvoll und notwendig ist, werden die Darstellungen in Kapitel 5 und 6 zeigen. 
einer bestimmten Art sein müssen. Das heißt, die einzelnen Verbbegleiter werden vom Verb sortal regiert (vgl. ebd. 390). Die sortalen Forderungen werden als Subskripte an den einzelnen Relatklammern notiert und sind als Abkürzungen entsprechender Bedeutungsregeln zu lesen.

Bezogen auf das Verb verstecken kann etwa die y-Relatstelle nur von einem belebten Individuum gefüllt werden, die x-Relatstelle muss durch etwas Konkretes realisiert werden und die s-Stelle ist für endliche Situationen reserviert. Derartige sortale Restriktionen wirken sich insofern auf die syntaktische Umgebung des verbalen Valenzträgers aus, „als die Prädikate, die zur Formulierung der Argumente dienen, keine mit den Forderungen in Konflikt stehenden Folgerungen beinhalten dürfen“ (ebd. 388):

(58) a. *dass der Schlüssel den Hausmeister versteckt.

b. DEN HAUSMEISTER [+konkret $\mathrm{x}$ ] \& DER SCHLÜSSEL [-belebty] \& VERSTECKT [+endlichS] ${ }_{+ \text {+konkret } \mathrm{X})}{ }_{\left.+{ }_{+} \text {belebt } \mathrm{Y}\right)}$

Entsprechend resultiert die Ungrammatikalität von (58a) - wie die Bedeutungskomposition in (58b) erkennbar werden lässt - aus dem Widerspruch zwischen der vom Verb an y gestellten Forderung nach Belebtheit und der Unbelebtheit des für x eingesetzten Argument-Prädikats.

Sortale Merkmalsforderungen entsprechen Präsuppositionen, die eingrenzen, „für welche Entitäten man überhaupt sinnvoll die Frage nach dem Zutreffen des Prädikats stellen kann“ (ebd.). Das zeigt sich u. a. daran, dass sie bei normaler Satznegation erhalten bleiben. So folgt sowohl aus Er erblickt die Tür als auch aus Er erblickt die Tür nicht die Belebtheit des Subjekts. Zu berücksichtigen ist jedoch, dass - anders als bei den bisher besprochenen Valenzbindungsbeziehungen - Verletzungen sortaler Forderungen nicht zwangsläufig zu inakzeptablen Sätzen führen. Formulierungen wie in (59) sind in einem metonymischen oder metaphorischen Sinn durchaus möglich:

(59) a. Der Schweinebraten an Tisch sieben möchte noch ein Bier. (nach Jacobs 2003: 289)

b. Deutschland trauert um seinen größten Rennfahrer. (ebd.)

„Wie solche [...] Verletzungen einer vom Verb ausgehenden sortalen Forderung zu explizieren sind, ist noch umstritten.“ (ebd. 389) Diskutiert werden Fälle wie (59a) vor dem Hintergrund vom syntaktischen Kontext ausgelöster Uminterpretationen der Argumente (,coercions‘, vgl. Pustejeovsky 1995); in Sätzen wie (59b) werden hingegen lexikalische Polysemie des Subjekt-Nomens bzw. ein unterspezifizierter Lexikoneintrag des Verbs diskutiert (vgl. ebd). 


\section{Rollenforderung}

Neben sortalen Forderungen unterliegen Relatpositionen häufig auch sog. Rollenforderungen, d. h. „Festlegungen der Weise, wie das Relat in die vom jeweiligen Ausdruck beschriebene Situation involviert ist“ (Jacobs 2003: 389). Die Subjekt-Relatposition von verstecken fordert neben Belebtheit (vgl. sortale Spezifikation) auch, dass die entsprechende Entität die beschriebene Situation kontrolliert, also die Fähigkeit besitzt, „die Handlung selbstständig einzuleiten oder abzubrechen sowie die Fähigkeit [sic!] sie selbstständig durchzuführen“ (Primus 1998: 116).

Kontrolle ist neben weiteren Eigenschaften, die u. a. in Blume 2000 entwickelt werden, für die als Agens bezeichnete semantische Rolle charakteristisch. Allerdings sind nicht alle typischen Eigenschaften für das Vorliegen eines Agens auch notwendig. Vielmehr handelt es sich bei dem Konzept des Agens im Sinne Dowtys (1991) um eine Art Prototypen-Begriff ${ }^{66}$, „der in dem Maß zutrifft, in dem einzelne für ihn charakteristische Eigenschaften vorliegen“ (Jacobs 2003: 389) eine Beobachtung, die gleichermaßen für die Patiens-Rolle und ihre charakteristischen Eigenschaften gilt.

Bei Rollenforderungen handelt es sich - anders als bei sortalen Merkmalsforderungen - i.d.R. um Implikationen, die von normaler Satznegation betroffen werden: So folgt zwar aus Er erblickt die Tür die Situationskontrolle des Subjekts, aus Er erblickt die Tür nicht jedoch nicht.

Valenzrelevant werden Rollenanforderungen zum einen dadurch, dass sie ähnlich den sortalen Forderungen - der syntaktischen Umgebung des Verbs inhaltliche Restriktionen auferlegen. Entsprechend sind Sätze unakzeptabel, die gegen die vom Valenzträger ausgehenden Rollenforderungen des Valenzträgers (hier: Handlungskontrolle) verstoßen:

^Der schlafende Polizist versteckt den Schlüssel.

Rollenforderungen sind für die Valenztheorie auch bedeutsam, weil „die lexikalischen Regeln, die die semantische mit der kategorialen Valenz verknüpfen, wesentlich auf solche Forderungen Bezug nehmen“ (ebd. 390). Ein Faktor, der

66 Im Rahmen dieser Arbeit wird die Idee eines mehrdimensionalen, auf Ähnlichkeitsbeziehungen basierenden Proto-Rollen-Ansatzes vertreten, wie er u. a. von Lakoff (1977) und Dowty (1991) eingeführt wurde. Die dabei angenommenen Proto-Rollen-Dimensionen ermöglichen es, spezifischere semantische Rollen zu definieren und z. B. als agens- oder patiensähnlich auszuweisen.

In der Forschungsliteratur sind unterschiedliche Ausprägungen dieser Idee zu finden. In der vorliegenden Arbeit wird der an Dowtys Ansatz orientierte Proto-Agens-Begriff nach Primus (2012a) zugrunde gelegt. 
sich besonders bei den durch die entsprechenden Rollenforderungen gesteuerten Kasusforderungen an die Argumente zeigt.

Um Rollenforderungen, d. h. syntagmatische Beziehungen der Partizipation im Sinne von Jacobs (ebd.), zu repräsentieren, werden sie analog zu den sortalen Forderungen als Subskripte an den einzelnen Relatpositionen vermerkt (vgl. (61a)). Auch sie sind als Abkürzungen entsprechender Bedeutungsregeln (61b) aufzufassen:

(61) a. SR: VERSTECKEN [s] (x) (+belebt, +Kontrolley)

b. BR: $\forall \mathrm{x} \forall \mathrm{y} \forall \mathrm{s}$ [VERSTECKEN (x) (y) [s] $\rightarrow$ KONTROLLE(y) (s)]

Nicht unproblematisch ist jedoch

auch nach der Wende zu einer flexibleren Analyse, bei der Agens und Patiens als ProtoRollen aufgefasst werden, die Frage, mit welchem Inventar konkreter Rollenforderungen (wie Kontrolle, Verursachung, Wahrnehmung) man die Zusammenhänge zwischen semantischer und kategorialer Valenz in natürlichen Sprachen am besten erfasst.

(ebd.)

\section{Repräsentationsformat semantischer Valenzforderungen}

Aus den vorangegangenen Ausführungen ergibt sich für die semantische Valenz (im Anschluss auch SemVal) folgendes, im weiteren Verlauf der Arbeit zugrundegelegtes Repräsentationsformat, vgl. exemplarisch für das Verb verstecken $(62)^{67}$ :

(62) SemVal: $\lambda x \lambda y \lambda s$ [VERSTECKEN $\left.[\mathrm{s}]\left({ }_{+ \text {konkret }} \mathrm{X}\right)\left(_{+ \text {belebt, }+ \text { Kontrolle }} \mathrm{Y}\right)\right]$

Die semantische Valenz wird durch die präfigierten Lambda-Variablen-Paare zum Ausdruck gebracht (vgl. Jacobs 1994b: 289). Sie geben an, dass die Bedeutung von verstecken ein dreistelliges Prädikat ist. Die Variablen in den Klammerpaaren markieren dabei die Relate der durch das Prädikat denotierten Beziehung, wobei $\mathrm{x}$ und $\mathrm{y}$ für Individuen oder Objekte stehen und s für eine Situation. Während dem Situationsrelat keine kategoriale Valenzstelle entspricht, repräsentiert die erste Individuenrelatposition der semantischen Valenz von verstecken die Stelle ,/akk' in der kategorialen Valenz ,/nom/akk des Valenzträgers, die zweite Individuenrelatposition die Stelle ,/nom'. Diese Korrespondenzen werden durch Indizierungen in der kategorialen Valenz (vgl. (51)) sichtbar gemacht.

67 Im Weiteren wird auf die Realisierung einer Situationsvariablen verzichtet, da sie - wie bereits erläutert - für die Untersuchungen der vorliegenden Arbeit nicht relevant ist. 
Darüber hinaus werden in der semantischen Valenz die mit einem Valenzträger einhergehenden Interpretationsbesonderheiten - d.h. u.a. die sortalen Restriktionen und Rollenforderungen - erfasst; sie werden durch entsprechende Subskripte wiedergegeben. Ausbuchstabieren lassen sich diese Besonderheiten für beliebige Argumente als Bedeutungsregeln (vgl. Jacobs 1994b), s. (63):

$$
\forall \mathrm{x} \forall \mathrm{y} \forall \mathrm{s} \text { [VERSTECK }[\mathrm{s}](\mathrm{x})(\mathrm{y}) \rightarrow \operatorname{KONKRET}(\mathrm{x}) \& \operatorname{BELEBT}(\mathrm{y}) \& \operatorname{KONTROLLE}(\mathrm{y})]
$$

Die in diesen Regeln ausgedrückten Beschränkungen wirken sich derart auf die syntaktische Umgebung der Verben aus, als die bei der Äußerung von Sätzen gewählten Entitäten nicht im Widerspruch zu diesen Forderungen stehen dürfen.

Auch die semantischen Valenzmerkmale werden im Folgenden nicht nur zur Darstellung der einzelnen Valenzträger genutzt; vielmehr dienen sie auch dazu, die zur Überprüfung von F2 zu unterscheidenden Verblesarten zu ermitteln (vgl. hierzu auch Kapitel 3.2). So konstatiert Primus, dass

die meisten Verben [...] mehrere miteinander verwandte Bedeutungen [haben, TvdB], die sich $u$.a. durch unterschiedliche semantische Valenzrahmen voneinander unterscheiden, so dass man von Lexemvarianten ausgehen muss.

(Primus 2015: 88 - FN 6)

Es zeigt sich, dass „die mit Wörtern [konkret: Verblesarten, TvdB] verbundenen Relatpositionen ein zentraler Aspekt ihrer Bedeutung“ (ebd. 386) sind. Die in (64) und (65) dargestellten singen-Verwendungen unterscheiden sich z. B. dadurch, dass bei singen im Sinne von jemand ist als Sänger tätig (vgl. E-VALBU_singen; 30.08.2018) automatisch eine Entität mitgedacht wird und zwar die desjenigen, auf den die Eigenschaft, Sänger zu sein, zutrifft. Dagegen sind bei singen im Sinne von jemand/etwas bringt etwas Melodisches mit seiner Stimme hervor (vgl. ebd.) zwei Relatpositionen bedeutungskonstitutiv: Zum einen die desjenigen, der die Tätigkeit des Singens ausführt, und zum anderen das, was singend hervorgebracht wird.

Beide Verbverwendungen sind darüber hinaus mit je spezifischen inhaltlichen Forderungen verbunden; das Singen als Sängerin, also die gesangliche Interpretation von Musikstücken, ist z. B. nur für Menschen möglich, vgl. (64), wohingegen auch Tiere - etwa Papageien - etwas Melodisches mit ihrer Stimme hervorbringen können, vgl. (65):

(64) singen

i.S.v. $\quad$ jemand ist als Sänger tätig

Bsp.: Was hat sie für einen Beruf? Sie singt. (ebd.)

SemVal: $\lambda \mathrm{x}\left[\operatorname{SING}\left({ }_{+ \text {Mensch }} \mathrm{x}\right)\right]$ 
(65) singen

i.S.v. jemand/etwas bringt etwas melodisches mit seiner Stimme hervor

Bsp.: $\quad$ Wir haben heute gefeiert und Lieder gesungen. (ebd.)

SemVal: $\quad \lambda x \lambda y\left[\operatorname{SING}\left({ }_{+ \text {Lied }} \mathrm{x}\right)\left(_{+ \text {belebt }} \mathrm{y}\right)\right]$

Welche Verfahren zur Ermittlung (lesartenspezifischer) Relatpositionen sowie damit einhergehender sortaler Restriktionen und Rollenforderungen in der vorliegenden Arbeit zum Einsatz kommen, wird in Kapitel 4 ausführlich dargestellt.

\subsection{Fakultative Ergänzungen}

Komplementweglassung ist im Deutschen zweifelsohne durch die Verbwahl beschränkt. Manche Verben erlauben sie, andere blockieren sie unter sonst gleichen Bedingungen, vgl. (1) - hier wiederholt in (66):

(66) a. Peter isst (einen Hamburger).

b. Peter verspeist *(einen Hamburger).

Weglassbare Komplemente - sog. fakultative Ergänzungen - bereiten älteren, zumeist monokriterial ausgerichteten Valenzansätzen große konzeptuelle Schwierigkeiten. So ist lange Zeit unklar, ob Nicht-Weglassbarkeit nicht schon aus der Definition valenzgeforderter Komplemente (bzw. Ergänzungen) folgt. Neueren multidimensionalen Auffassungen von Valenz bereiten sie hingegen weniger Probleme. Der Nutzen solcher Valenzauffassungen besteht nicht in der bloßen Auflistung unterschiedlicher Valenzdimensionen, sondern in der Feststellung, dass die verschiedenen Valenzdimensionen trotz einzelner (unilateraler) Implikationsverhältnisse (vgl. Jacobs 1994a) unabhängig voneinander sind. Es handelt sich also nicht um verschiedene Aspekte ein und desselben Phänomens, die immer gemeinsam vorliegen müssen, sondern um verschiedene Phänomene, die - im prototypischen Fall - zwar gleichzeitig vorliegen können, aber nicht müssen (vgl. Jacobs 2003: 390ff.). In diesem Sinne lassen sich mit Hilfe eines multidimensionalen Valenzkonzepts Verbbegleiter sehr differenziert nach ihrer jeweiligen Beurteilung in den verschiedenen Valenzdimensionen ordnen. So gilt eine „Konstituente X, die in einer dieser Relationen zu einer Konstituente Y steht, als von Y valenzgebunden oder als Ergänzung von Y“ (ebd. 391).

Die Unabhängigkeit der verschiedenen Valenzdimensionen zeigt sich darin, dass es sowohl Fälle globaler Divergenzen von kategorialer und semantischer Valenz gibt als auch solche, in denen einzelne Dimensionen der jeweils gleichen Valenzebene nicht übereinstimmen (vgl. ebd. 390). Bei den hier zu untersuchen- 
den fakultativen Ergänzungen kommt es zu Divergenzen auf gleicher - nämlich kategorialer - Valenzebene. In Sätzen wie in (67) scheinen Merkmals- ohne Realisierungsforderungen vorzuliegen:

(67) Peter sagt, dass er (den Vertrag) unterschreibt. (ebd. 393)

Nach der in der vorliegenden Arbeit vertretenen Valenzauffassung kann die Weglassbarkeit des Objekts in (67) jedoch nicht auf das Fehlen einer syntagmatischen Beziehung der Notwendigkeit im Sinne von „X ist in S für Y notwendig gdw. X in S einer Realisierungsforderung in der KR-Valenz von Y unterliegt“ (ebd. 390) zurückgeführt werden. Das Objekt von unterschreiben unterliegt der kategorialen Merkmalsforderung ,/akk' und damit notwendigerweise auch einer Realisierungsforderung (vgl. ebd. 393). So „wäre es unlogisch anzunehmen, eine Merkmalsforderung könne ohne Realisierung einer Konstituente, die das Merkmal hat, erfüllt werden“ (ebd.). Entsprechend verletzt (68b) für die kategoriale Valenzforderung ,/akk das Prinzip der syntaktischen Verarbeitung von Valenzmerkmalen, wonach diese durch eine Schwesterkonstituente gesättigt oder an die Mutterkonstituente transferiert werden müssen:

(68) a.

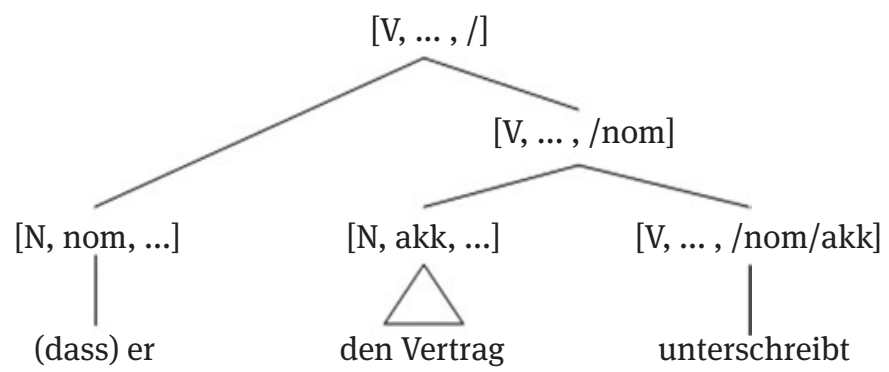

b.

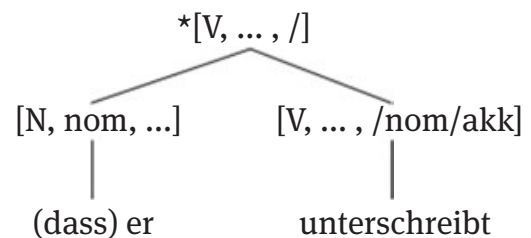

Eine mit diesen Überlegungen kompatible Erklärung für die Weglassbarkeit des valenzgeforderten Objekts in (67) wurde von Jacobs (1994b, 2003) entwickelt. Hiernach liegen bei fakultativen Ergänzungen lexikalische Variantenbildungen hinsichtlich der kategorialen Valenzmerkmale vor. In diesem Sinne liegt der „Weglassung einer fakultativen Ergänzung [...] die Wahl einer vom Lexikon bereit- 
gestellten reduzierten syntaktischen Valenz (R-Valenz) und einer entsprechend reduzierten syntaktischen Struktur zugrunde“ (Jacobs 1994b: 297).

Entsprechend ist das in (67) dargestellte Verb unterschreiben „to be represented as having two lexical variants, namely a non-reduced variant [...] and a reduced variant“ (Engelberg 2002: 373). Es weist somit neben der kategorialen Valenz ,/nom /akk', vgl. (69a), eine weitere, um eine Stelle reduzierte kategoriale Valenz auf, vgl. (69b):

(69) unterschreiben
a. /nom /akk
b. /nom

Durch den Wegfall der Merkmalsforderung ,/akk in (69b) wird auch die Realisierung eines Akkusativdependens nicht mehr gefordert. Entsprechend erfüllt die in (70) dargestellte Struktur alle zuvor besprochenen Prinzipien.

(70)

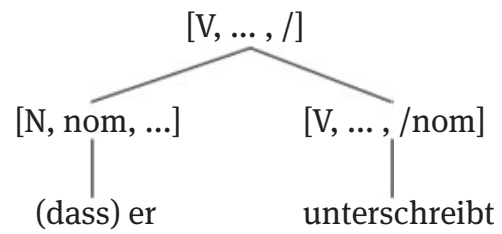

Wenn unterschreiben neben einer zweistelligen auch eine einstellige syntaktische Valenz hat, weist es neben einer zweistelligen auch eine einstellige Bedeutung auf (vgl. Jacobs 1994b: 298). Dabei muss jedoch der Tatsache Rechnung getragen werden, dass „bei Aktivierung der R-Valenz [...] das syntaktisch nicht mehr vorhandene Objekt implizit - d.h. in der Bedeutungsstruktur - durchaus noch präsent ist“" (ebd.). So wird auch in der objektlosen Verwendung des Verbs in (70) ein Objekt mitverstanden in dem Sinne, dass der Vorgang des Unterschreibens nur stattfindet, wenn es etwas Konkretes, z. B. ein Schriftstück, gibt, das unterschrieben wird. Diesen Umstand kann man mit dem unter Punkt 2.3.2.2 angedeuteten Repräsentationsformat impliziter Relate erfassen, „d.h. dadurch, dass in der entsprechenden SR eine Objekt-Position angenommen wird, der keine Position in der KR-Valenz entspricht“ (Jacobs 2003: 393), vgl. (71) für die intransitive Verwendung von unterschreiben:

\section{(71) SR: UNTERSCHREIB (x) (y)}

Damit sind abweichend zur syntaktischen Struktur in der „semantischen Repräsentation [...] beides [d.h. sowohl die transitive Voll- als auch die intransitive 
Reduktionsvariante, TvdB] zweistellige Verben, so daß die syntaktisch intransitive Variante über ein implizites Argument verfügt“ (Engelberg 1997: 11).

Die Art, wie ein solches implizites Argument interpretiert werden muss, hängt nicht allein von der Verwendungssituation ab, sondern unterliegt grammatischen Restriktionen. So ist schon oft beobachtet worden, dass Komplementweglassung bei machen Verben nur möglich ist, wenn das Objekt-Relat definit, d. h. in der Äußerungssituation bekannt ist. Derartige Forderungen können als Subskript in der semantischen Valenz angezeigt werden:

SR: UNTERSCHREIB ( $\mathrm{x})\left({ }_{+}\right.$DEF $)$

Damit wird nach Ansicht zahlreicher Autoren „die Annahme, in der objektlosen Verwendung [...] liege eine eigene Variante des Verbs ${ }^{68}$ vor, auch semantisch gestützt, denn für explizite Argumente von unterschreibt gibt es keine Definitheitsforderung [...]“ (Jacobs 2003: 393; Fußnote ergänzt, TvdB).

Einige Linguisten sehen in solchen Interpretationsbesonderheiten impliziter Argumente sogar den Hauptgrund für eine lexikalische Variantenbildung bei Komplementweglassung (vgl. Engelberg 2002: 373). Ihrer Ansicht nach sind „Verben mit fakultativen Ergänzungen in den objektlosen Verwendungen stets mit spezifischen Bedeutungen verbunden“ (ebd.) ${ }^{69}$. Daher gilt, dass die

reduced variants of verbs are characterized by semantic peculiarities which do not hold for the non-reduced variant. In particular, in most cases the interpretation of an implicit argument underlies restrictions that are stronger than the selectional-restrictions which the transitive variants impose on the respective non-implicit argument. (Engelberg 2002: 373)

Spezialisierte implizite Ergänzungen zeichnen sich dabei „nicht nur durch ihre geringere sortale Variationsbreite [aus, TvdB], sondern auch dadurch, daß sie einer semantisch von der Normalvariante unterschiedenen Verbvariante zugeordnet sind“ (Jacobs 1994b: 302). In diesem Sinne wird für entsprechende Verben davon ausgegangen, dass die unterschiedlichen Valenzvarianten ,,are represented by different predicate constants [...] each of which imposes particular restrictions upon

68 Hiermit ist nicht die Herausbildung eines neuen Lexikoneintrags im Sinne eines eigenen Lemmas gemeint. Vielmehr liegt hier lexikalische Variantenbildung vor, wobei beide Valenzvarianten - d. h. sowohl die transitive als auch die intransitive - dem gleichen Verb, besser der gleichen Verblesart (s. Kapitel 5 und 6), zuzuordnen sind.

69 Um welche Interpretationsbesonderheiten es sich im Einzelnen handelt, wird in den Kapiteln 5 - mit Blick auf semantische Spezialisierungen impliziter Argumente - und in Kapitel 6 - mit Blick auf die Unterscheidung zwischen definiten und indefiniten Weglassungen - thematisiert. 
its arguments“ (Engelberg 2002: 374). Solche Beschränkungen werden i.d.R. als Interpretationsbesonderheiten notiert und in Bedeutungsregeln ausbuchstabiert.

Für das Verb unterschreiben können demzufolge die in (73) dargestellten Varianten unterschreiben - transitive Verbverwendung - und unterschreiben ${ }_{R}-$ (valenzreduzierte) intransitive Verbverwendung - angenommen werden ${ }^{70}$ :

\author{
a. unterschreiben \\ synVal: $/$ nom $^{\mathrm{y}} / \mathrm{akk}^{\mathrm{x}}$ \\ semVal: $\lambda x \lambda y$ [UNTERSCHREIB $(\mathrm{x})(\mathrm{y})]$ \\ b. unterschreiben ${ }_{R}$ \\ synVal: $/$ nom $^{\mathrm{y}}$ \\ semVal: $\lambda y$ [UNTERSCHREIB $\left.{ }_{\mathrm{R}}\left(+{ }_{+D E F} \mathrm{x}\right)(\mathrm{y})\right]$ \\ BR: $\quad \forall \mathrm{x} \forall \mathrm{y}$ [UNTERSCHREIB ${ }_{\mathrm{R}}(\mathrm{x})(\mathrm{y}) \rightarrow$ IN S BEKANNT $\left.(\mathrm{x})\right]$
}

Bei (73b) handelt es sich um die einer Weglassungsoption entsprechende stellenreduzierte Alternativvalenz (R-Valenz). Sie weist ein nicht- $\lambda$-gebundenes, damit implizites Argument auf (vgl. Engelberg 2002: 373) und ist im Vergleich zur nichtstellenreduzierten Valenz derselben Lesart (vgl. (73a)) mit Interpretationsbesonderheiten - hier in Form einer definiten Interpretation des impliziten Arguments verbunden $^{71}$.

70 In der Literatur werden die beiden Valenzvarianten häufig durch eine numerische Indizierung voneinander unterschieden (z. B. transitives unterschreiben $\rightarrow$ unterschreiben ${ }_{1}$; (valenzreduziertes) intransitives unterschreiben $\rightarrow$ unterschreiben $_{2}$ ) (vgl. z. B. Engelberg 1997).

Da im weiteren Verlauf der Arbeit jedoch unterschiedliche Verblesarten mit Hilfe arabischer Ziffern voneinander unterschieden werden (vgl. insb. die Darstellungen in Kapitel 5), werden im Folgenden valenzreduzierte Verbverwendungen durch ein indiziertes $<R>(=R$-Valenz) ausgewiesen. Um die Lesartenabhängigkeit entsprechender Angaben zu erfassen, wird zusätzlich durch einen Unterstrich abgetrennt - die Lesart, auf die sich die R-Valenz bezieht, angegeben (z. B. unterschreiben ${ }_{5 \_}$).

71 Um die Interpretationsbesonderheiten impliziter Argumente, d.h. den Wechsel der Selektionsrestriktionen von der transitiven zur intransitiven Verbverwendung zu erfassen, wurden verschiedentlich auch pragmatische Erklärungsansätze herangezogen. Danach verweisen intransitive Verbverwendungen immer auf eine Art Standardsituation (vgl. Engelberg 2002: 374).

Die Unzulänglichkeiten pragmatischer Erklärungsansätze zeigen sich nun u.a. darin, dass eine solche Annahme bei vielen Verben gerade nicht zutreffend ist. So wird z. B. intransitiv verwendetes geben in einem Satz wie Sie gab immer interpretiert im Sinne von Sie gab den Mitspielern (Spiel-) Karten (vgl. Jacobs 2003: 393). Ein solcher Satz beschreibt jedoch keine standardisiertere Situation als das Geben in einem Satz wie Sie gab den Kindern Bonbons (vgl. Engelberg 2002: 374).

Im Sinne eines pragmatischen Erklärungsansatzes wäre nun zu erwarten, dass in einem komplementlosen Satz mit geben eine solche Bonbon-geben-Interpretation ebenfalls verfügbar 
Die enge semantische Verbindung zwischen den beiden Verbverwendungen kann durch eine Bedeutungsregel wie in (74) erfasst werden (vgl. ebd.):

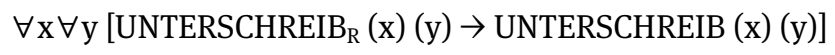

Nachfolgend wird es vor allem darum gehen, die für implizite Argumente angenommenen und in der Literatur beschriebenen semantischen Spezialisierungen $\mathrm{zu}$ beschreiben und kritisch zu reflektieren. Dazu werden in Kapitel 5 umfangreiche Untersuchungen durchgeführt sowie deren Ergebnisse dargestellt. Daran schließen sich in Kapitel 6 Überlegungen an zur Unterscheidung definiter und indefiniter impliziter Argumente.

Im Vordergrund aller Untersuchungen steht dabei die Frage, inwieweit Interpretationsbesonderheiten tatsächlich eine (natürliche) Folge von Komplementweglassungen sind (vgl. Jacobs 1994b; 2003). Möglich wäre auch, sie auf eine schon vor Argumentweglassung bestehende Lesartendifferenzierung zurückzuführen. Bei den einzelnen Verblesarten handelt es sich dann um bedeutungsverschiedene Valenzträger mit alternierenden, je spezifischen Valenzmustern. Eine stellenreduzierte Alternativvalenz wäre dann einer dieser Verblesarten zuzuordnen, der sie im Vergleich mit der nicht-stellenreduzierten Valenz keine Interpretationsbesonderheiten hinzufügt. Vielmehr übernimmt sie die sortalen Eigenheiten der jeweiligen Verblesart, die Weglassung wäre somit sortal-semantisch neutral.

Zur Beantwortung dieser Frage muss zunächst das Verwendungsweisenspektrum der hier zu untersuchenden Verben ermittelt werden. Daher schließen sich in Kapitel 3 zunächst Ausführungen zu unterschiedlichen Positionen im Umgang mit Bedeutungsvarianten sprachlicher Ausdrücke an, bevor in Kapitel 4 nach einer Möglichkeit gesucht wird, die verschiedenen Lesarten der hier zu untersuchenden Verben nach objektivierbaren Kriterien zu ermitteln.

ist. Dies ist jedoch nicht der Fall. Eine solche Interpretation ist nicht einmal verfügbar, wenn die Kinder die (anderen) Teilnehmer des Kartenspiels sind und als solche Karten und Süßigkeiten erhalten (vgl. ebd.). 